EM

\title{
AGROPECUÁRIA E DESMATAMENTO NA AMAZÔNIA LEGAL BRASILEIRA: uma análise espacial entre 2007 e 2017
}

\section{AGRICULTURE AND DEFORESTATION IN BRAZILIAN LEGAL AMAZON: a spatial analysis between 2007 and 2017}

Roger Alexandre ROSSONI ${ }^{1}$ Marcelo Lopes de MORAES ${ }^{2}$

\begin{abstract}
Resumo: O artigo tem como objetivo analisar a distribuição e a concentração espacial do desmatamento e das atividades agropecuárias, representadas pela área de lavoura e o efetivo de pecuária, nos municípios da Amazônia legal, entre 2007 e 2017. Com base nos dados de desmatamento do INPE, foram calculados os indicadores de I de Moran, com o intuito de verificar a existência de efeitos espaciais no avanço do desmatamento, bem como sua relação com a agropecuária. Os resultados indicam que o avanço do desmatamento ocorreu de forma mais intensa em uma faixa de área que abrange o Acre, Sul do Amazonas, Pará e Amapá, assim como Roraima. Por meio da análise do I de Moran global, constatou-se a existência de concentração espacial do desmatamento. Apesar de não identificar efeitos espaciais globais significativos entre desmatamento e pecuária, ao analisar de forma local, foram identificadas áreas com presença de correlação estatisticamente significante.
\end{abstract}

Palavras-Chave: Floresta; Moran; Brasil; Agricultura; Pecuária.

\begin{abstract}
This paper aims to analyze the distribution and spatial concentration of deforestation and farming activities, represented by the area of agriculture and livestock in the municipalities of the legal Amazon, between 2007 and 2017. The Moran's I indicators were calculated, based on the INPE deforestation data, with the purpose of verifying the existence of spatial effects on the progress of deforestation, as well as its relations with agriculture and livestock. The results indicate that the advance of deforestation occurred intensely in an area that embraces Acre, Southern Amazonas, Pará and Amapá, as well as in Roraima. Through the analysis of the global Moran's I, it was verified the existence of spatial concentration of deforestation. Despite not identifying significant global spatial effects between deforestation and livestock, when analyzing locally, areas with statistically significant correlation were identified.
\end{abstract}

Keywords: Florest; Moran; Brazil; Agriculture; Livestock.

\section{Introdução}

A Amazônia legal brasileira é composta por $5.020 .000 \mathrm{~km}^{2}$, distribuídos entre nove unidades federativas e 774 municípios (Mapa 1). O território amazônico apresenta uma grande complexidade dadas suas características singulares. Entre elas, a predominância da área de floresta, acompanhada da fragilidade socioeconômica de parte significante de sua população.

\footnotetext{
${ }^{1}$ Mestre em Gestão e Desenvolvimento Regional e professor da Universidade Tecnológica Federal do Paraná (UTFPR) - roger.rossoni@gmail.com

${ }^{2}$ Doutor em Economia Aplicada e professor da Universidade Estadual do Oeste do Paraná (UNIOESTE) marcelomoraes.unioeste@gmail.com
} 
Mapa 1 - Amazônia Brasileira - Biomas.

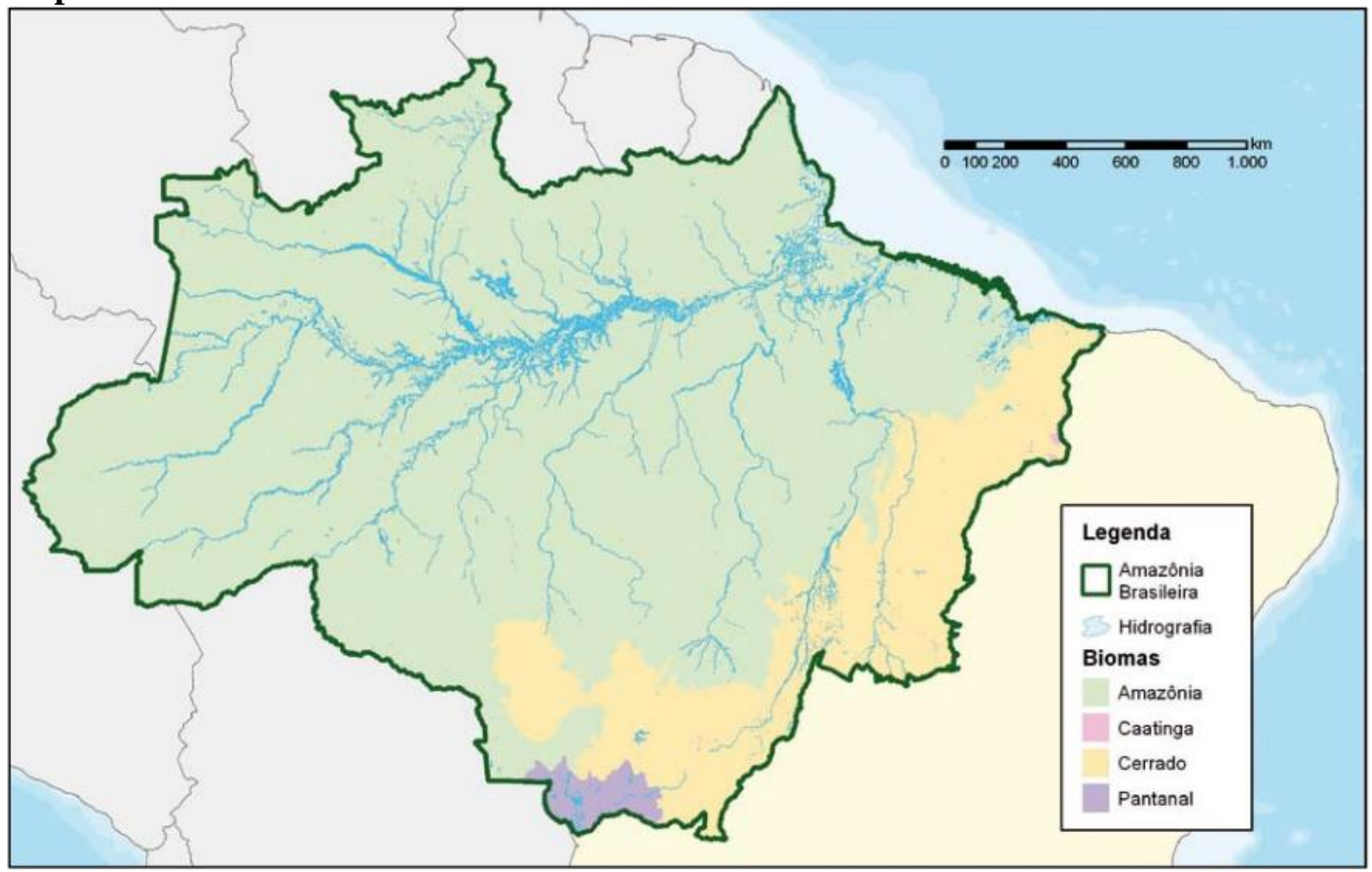

Fonte: Brasil, 2008.

A crescente preocupação com questões ambientais, como o desmatamento e o aquecimento global, bem como a persistência da pobreza, auxiliou a introduzir a Amazônia como foco de pesquisa de diversas áreas de conhecimento a nível internacional.

O princípio do desmatamento da Amazônia brasileira, em larga escala, está associado ao programa de integração territorial, via rodovia transamazônica, na década 1970 , e a ocupação do território amazônico (FEARNSIDE, 2009). Desde então, o desmatamento ampliou-se de forma significante.

O aumento do desmatamento da Amazônia brasileira, a partir de 1988, primeiro ano com dados disponíveis no Instituto Nacional de Pesquisas Espaciais (INPE), é apresentado no Gráfico 1. Verifica-se uma redução no avanço do desmatamento após 2003. Possivelmente, tal resultado está associado à aplicação de políticas mais abrangentes, compreendendo o aumento do monitoramento e repressão ao desmatamento (ASSUNÇÃO, GANDOUR \& ROCHA, 2015), e a intervenção na cadeia produtiva da soja e da pecuária (NEPSTAD et al., 2014). Contudo, ressalta-se que o desmatamento cresce continuamente no período analisado.

A dificuldade em enfrentar o desmatamento está associada à complexidade de sua dinâmica. Além da ocupação territorial, fatores como os subsídios fiscais, a atividade agropecuária e o valor das terras são comumente citados como fatores relacionados ao desmatamento (MAHAR, 1989; MARGUILIS, 2003; FEARNSIDE, 2005).

A característica multifatorial do desmatamento também é notada em seus efeitos. O mais irrefutável dos efeitos é a depredação do bioma. Trabalhos como Sodhi (2004), Brock, Shodi; Ng (2003), Skole e Tucker (1993) apontam que o desmatamento de florestas do trópico úmido está diretamente associado ao processo de extinção de espécies e da redução da biodiversidade. Esse processo não se limita às áreas desmatadas, mas também a áreas contíguas.

Além da mudança no bioma amazônico, o aumento do desmatamento pode ocasionar o aumento de temperatura e a ampliação da estação de seca, dificultando a restauração da 
floresta (SHUKLA, NOBRE, SELLERS, 1990). Medvigy, Walko, Avissar (2011) apontam que, além de reduzir o volume de chuvas, o desmatamento, possivelmente, está relacionado à alteração em sua distribuição espacial, tornando o Noroeste da Amazônia mais seco e o Sudeste mais chuvoso. Além de alterar a distribuição espacial da precipitação, a ocorrência de eventos extremos de frio, em relação à temperatura local, se tornara mais frequente.

Gráfico 1 - Evolução do Desmatamento na Amazônia Legal Brasileira, em Quilômetro Quadrado $\left(\mathrm{km}^{2}\right)$, e a Área Acumulada de Desmatamento de 1988 a 2018*.

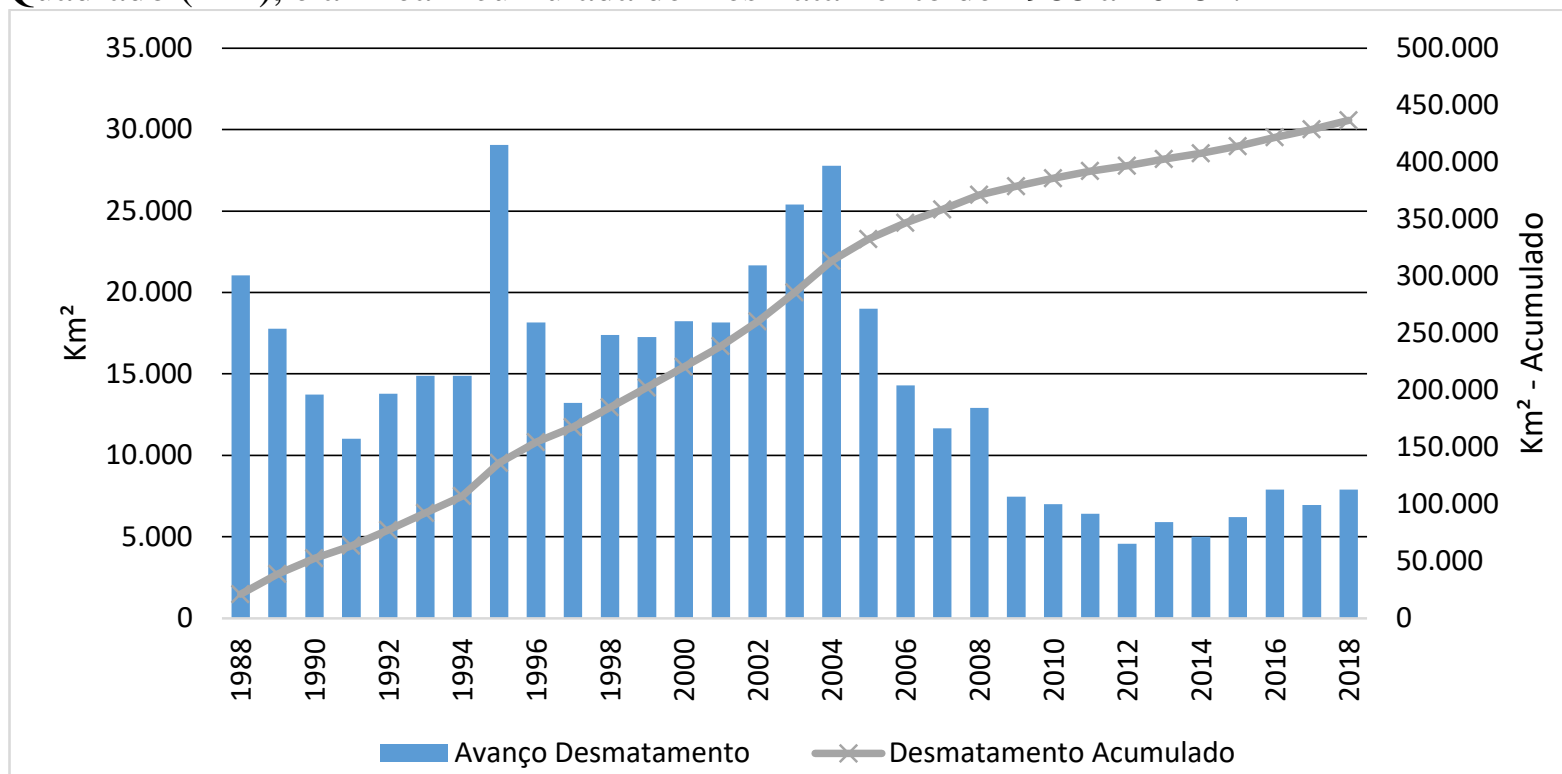

* O desmatamento em 1988 equivale à média do desmatamento da Amazônia Legal de 1977 a 1988 ; o desmatamento nos anos de 1993 e 1994 é a média entre esses dois anos; e o desmatamento em 2018 representa uma estimativa, enquanto os demais anos são dados consolidados. O desmatamento acumulado é o somatório do desmatamento, não considerando áreas desmatadas que foram reflorestadas.

Fonte: INPE (2019).

Org: Elaborado pelos autores.

Os impactos do desmatamento não se limitam ao aspecto ambiental, mas incluem aspectos socioeconômicos, como a restrição de possibilidades de desenvolvimento econômico sustentáveis, com base nos ativos locais. Conforme Diniz et al., (2008), a existência da armadilha da pobreza no território da Amazônia brasileira dificulta o processo de crescimento econômico. A exploração da base de recursos naturais não consegue superar a situação de pobreza e, por fim, extingue tal potencial de desenvolvimento econômico

Desse modo, a fragilidade socioeconômica não pode ser associada à escassez de recursos, e sim à dificuldade de exploração de forma sustentável, a qual pode ser considerada uma alternativa de geração de produção e renda. Destaca-se a existência de um trade-off entre a preservação da floresta e o desmatamento para atividades alternativas. Os resultados de Marguils (2003), ao estimar os custos de oportunidade do desmatamento e de alternativas sustentáveis, sugerem que os ganhos econômicos privados do desmatamento e atividades realizadas após o desmatamento, superam os ganhos de sua preservação. De modo complementar, Chagas e Andrade (2017) demonstram que o custo de preservação ambiental é maior quanto menor for o retorno imediato, por meio da extração dos recursos naturais, em relação ao rendimento de longo prazo, por meio da atividade agropecuária. Ambos os resultados indicam a necessidade de criar mecanismos de mercado ou de compensação global para que o ganho social, por meio dos serviços florestais, seja efetivamente incorporado à decisão econômica financeira de manutenção da floresta. 
Apesar do relativo sucesso da redução do desmatamento a partir do século XXI, a construção de uma alternativa sustentável, agregando ganhos econômicos, ambientais e sociais continua sendo um desafio. Ao passo que não se concretiza uma resolução sustentável, o avanço da agropecuária impulsiona o aumento da pressão ambiental, reforçando o processo de desmatamento (DINIZ et al., 2008) (DINIZ et al., 2009) (RIVERO et al., 2009).

Considerando a persistência do desmatamento na Amazônia brasileira e suas diversas implicações, o presente estudo tem como objetivo analisar a distribuição e a concentração espacial do desmatamento e das atividades agropecuárias, representadas pela área de lavoura e o efetivo de pecuária, nos municípios da Amazônia legal entre 2007 e 2017.

Com base nos dados de desmatamento do INPE, foram calculados os indicadores de I de Moran, univariada e bivariada, global e local, com o intuito de verificar a existência de efeitos espaciais no avanço do desmatamento, bem como sua relação com a agropecuária.

Além da presente introdução, o artigo está estruturado do seguinte modo: Revisão de Literatura - apresenta alguns estudos relacionados ao desmatamento da Amazônia; Metodologia - discorre sobre os métodos aplicados e as variáveis utilizadas; Resultados apresenta análise dos resultados obtidos pela pesquisa; e, por fim, as Considerações Finais.

\section{A Economia e o Meio Ambiente: Uma Breve Revisão}

A ciência econômica analisa os impactos da atividade humana sobre o meio ambiente, seja pela agricultura ou pela indústria, dentro do arcabouço denominado Economia do Meio Ambiente. Costa (2005) descreve que a Economia do Meio Ambiente (ou Economia Ambiental) é uma área da economia que analisa as questões de manejo e preservação sob a ótica da teoria econômica. Já Melo (2005) descreve que o meio ambiente, para a ciência econômica, é um meio para a satisfação das necessidades humanas, já que este possui funções no desempenho da vida humana.

Costa (2005) cita três funções básicas oriundas das atividades econômicas (consumo e produção) sob meio ambiente: a oferta de serviços diretos ao consumo, como o ar e a água; o provimento de insumos para a produção, como as matérias primas; e o 'ambiente' de descarte dos resíduos derivado da produção e do consumo das famílias. No entanto, tais funções não são dissociadas e podem apresentar conflito, como o caso da água de um rio, a qual serve de consumo e pode, também, ser receptora de resíduos de uma indústria, afetando a sua função de ofertar diretamente ao consumo. Assim, os recursos naturais são escassos e apresentam uso alternativo, sendo um problema econômico a alocação eficiente desses recursos.

Entretanto, sob a ótica acadêmica, há uma linha que discorda sobre a escassez dos recursos naturais. Romeiro (2001) descreve que há duas correntes sobre a economia do meio ambiente no debate acadêmico: economia ambiental e economia ecológica. A Economia Ambiental, baseada na economia neoclássica, considerava a infinitude de recursos naturais, já que apenas capital e trabalho estavam especificados na função de produção. Assim, os recursos naturais não representavam, no longo prazo, um limite à expansão da economia. Após essa análise inicial, os recursos naturais foram incluídos na função de produção, mas tendo em conta que o sistema econômico é considerado "suficientemente grande para que a disponibilidade de recursos naturais $(\mathrm{RN})$ se torne uma restrição à sua expansão, mas uma restrição apenas relativa, superável indefinidamente pelo progresso científico e tecnológico" (ROMEIRO, 2001, p. 9).

Considerando uma função de produção $\mathrm{Y}=f(\mathrm{~K}, \mathrm{~L}, \mathrm{R})$, o autor explica que, desde que a quantidade de capital (K) seja suficientemente grande, a quantidade de recursos naturais $(\mathrm{R})$ pode ser tão pequena quanto se deseja. Ou seja, esgotada uma base de recursos, o sistema 
econômico se move suavemente para outra, não limitando o crescimento da economia no longo prazo. Essa substituição é garantida pela tecnologia e progresso científico.

A segunda corrente, representada pela Economia Ecológica, "vê o sistema econômico como um subsistema de um todo maior que o contém, impondo uma restrição absoluta à sua expansão" (ROMEIRO, 2001, p. 12). Ou seja, recursos naturais e capital são complementares e, compartilhando da mesma visão que a primeira corrente, considera-se o avanço tecnológico e científico como fundamental para elevar a eficiência no uso dos recursos naturais. Porém, discordam “...em relação à capacidade de superação indefinida dos limites ambientais globais" (p. 12). No longo prazo, sem a estabilização do consumo per capita, respeitando a capacidade de carga do planeta, não haverá sustentabilidade do sistema econômico.

Segundo Romeiro (2001), para essa segunda corrente, a questão central é considerar a existência desses limites no funcionamento da economia. Na primeira corrente, de abordagem neoclássica, esses limites não existem, devido à possibilidade de ajustes ilimitados, ou seja, o uso de recursos abundantes em substituição aos recursos que se tornaram escassos. Considerando bens ambientais transacionáveis em mercados, há um aumento do preço do bem cuja escassez é crescente, resultando na indução de inovações que irá poupá-lo e substituí-lo por outros bens naturais mais abundantes. Ressalta-se que os agentes supõem conhecer os estoques, o curso futuro do progresso tecnológico, as diferenças de qualidade e a demanda do uso desse bem ambiental que irá substituir o bem escasso.

Independente das divergências e convergências entre as linhas citadas, um dos principais temas a ser objeto de estudo no âmbito da Economia do Meio Ambiente é a relação dos custos e benefícios de se preservar a floresta ou desmatar. Tal relação é norteada pelas questões que afetam o desenvolvimento e a pobreza e, especificamente para o caso da Amazônia brasileira, o avanço da agropecuária apresenta-se como elemento central nessa análise.

Agropecuária, desmatamento e desenvolvimento

Há um debate sobre o avanço da agropecuária e a sua relação com o desmatamento e o desenvolvimento. Bacha (2004) explica que os modelos econômicos 'vigentes' em cada período inspiraram políticas desenvolvimentistas, as quais sempre foram associadas à destruição das florestas e uso insustentável dos remanescentes florestais. "As causas imediatas do desmatamento são a expansão da agropecuária, a criação de infraestrutura econômica (como estradas, hidrelétricas, barragens, por exemplo), a expansão urbana e a extração florestal" (BACHA, 2004, p. 394).

No que tange o desenvolvimento e a degradação ambiental, o Relatório de Bruntland discute essa causalidade, explicando que a pressão sobre os recursos naturais é decorrente das altas taxas de crescimento da população, maior dependência do setor agropecuário e maior parcela da população na zona de pobreza (WCED, 1987).

Sobre a expansão da agropecuária, Romeiro (2001) descreve que a agricultura pode preservar os equilíbrios ambientais fundamentais ao construir um ecossistema agrícola que conserve alguns mecanismos básicos de regulação ecológica, como a rotação de culturas e a manutenção da fertilidade do solo. Ou seja, é possível a inserção de um ecossistema "artificial", em detrimento de um ecossistema natural, com equilíbrio ecológico, o qual necessitará da ação humana nessa regulação ecológica.

Contudo, a degradação ambiental da Amazônia não pode ser interpretada como sinônimo de desenvolvimento. Pobreza, agropecuária e desmatamento são palavras-chave na Amazônia brasileira. Diniz et al., (2008) descrevem que a população dos estados que fazem parte da Amazônia Legal apresenta, em relação à média nacional, qualidade de vida muito 
baixa e alto nível de pobreza. Tal situação “[...] não reflete as várias transformações por qual vem passando a economia da região, ao longo das últimas três décadas" (DINIZ et al., 2008, p. 2). Tais transformações, refletidas em crescimento resultante da exploração dos recursos naturais, não alteram a pobreza crônica e são direcionadas por políticas estatais desenvolvimentistas.

Essa dificuldade em superar o problema por meio da exploração da base de recursos naturais está relacionada, entre outros fatores, com a fragilidade do Sistema Regional de Inovação da Amazônia. Tal fragilidade, resulta do baixo desempenho em termos de pesquisa científica e tecnológica, formação de capital humano e agregação de valor dos recursos locais (DINIZ, M. B., DINIZ, M. J. T., 2018).

Homma (2013) demonstra que, apesar dos avanços científicos, não houve transformação estrutural na região amazônica, persistindo a fragilidade socioeconômica. $\mathrm{O}$ autor destaca a necessidade de criar conhecimento de origem local, sendo que as políticas necessitam considerar, também, a inclusão do setor produtivo, não apenas a sustentabilidade ambiental. O aumento da produtividade da agricultura familiar da região amazônica pode reduzir a pressão sobre a exploração ambiental predatória.

Esforços para criar mecanismos de convergência entre desmatamento e desenvolvimento, em especial nas fronteiras de agricultura familiar, como na região Transamazônica, são de grande importância. Apesar da persistência do desmatamento, relacionado, principalmente, à pecuária, a diversificação do uso do solo gera um maior ganho para a população regional, em comparação com outras áreas de exploração não familiar. Desse modo, Alencar et al., (2004) indicam que essas áreas apresentam desmatamento socialmente mais aceitável.

O trade-off entre exploração ambiental no presente e a preservação de recursos, para potencial exploração futura, engloba uma população significativa com baixa qualidade de vida. Destarte, o desenvolvimento de uma alternativa econômica, social e ambientalmente sustentável é um desafio iminente.

\section{Estudos Correlatos}

A presente seção tem como objetivo apresentar estudos que abordam a distribuição espacial do desmatamento da Amazônia brasileira e sua relação com a atividade agropecuária.

Skole e Tucker (1993), por meio da análise computacional de imagens de satélite, mensuraram o desmatamento da Amazônia Brasileira e a degradação de sua biodiversidade entre 1977 e 1988. Os resultados demonstram o aumento de mais de $150 \mathrm{mil} \mathrm{km}^{1}$, área superior ao território da Grécia. Ao analisar a distribuição espacial do desmatamento, os autores indicam que o processo de desmatamento apresenta forte tendência de concentração espacial. Nota-se que o desmatamento se intensificou na margem Sul e Leste da Amazônia. Além da fronteira da floresta, verifica-se a expansão do desmatamento no interior da floresta, ao Leste do Amazonas e Centro do Pará.

Kampel, Câmara e Quintanilha (2000), por meio da Análise Exploratória de Dados Espaciais (AEDE), buscaram explorar as relações espaciais dos municípios da Amazônia legal, nos períodos de 1991 a 1992 e de 1992 a 1994, bem como possíveis relações com variáveis socioeconômicas. Ao analisar o I de Moran Global, verifica-se que houve aumento da concentração espacial da taxa de desmatamento, passando de 0,459 para 0,616 no período analisado. Em relação ao I de Moran local, identificou-se associação espacial positiva na região denominada "Arco" da Amazônia, abrangendo Leste do Acre, Rondônia, Norte do 
Mato Grosso e Sul do Pará. Como esperado, devido à breve amplitude temporal, os clusters de 1991 a 1992 e 1992 a 1994 são similares.

Rivero et al., (2009) examinaram a relação entre o uso do solo e o desmatamento na Amazônia Brasileira entre 2000 e 2006. Destaca-se que a expansão do desmatamento ocorreu de modo mais intenso no entorno da denominada "fronteira consolidada de desmatamento", região que abrange o Sudeste Paraense, o Sul do Mato Grosso, o Centro de Rondônia, o Leste do Maranhão e o Norte do Tocantins, e tem a maior parte da sua área já desmatada. Por meio de regressões lineares com dados em painel, demonstrou-se que o desmatamento está fortemente correlacionado com a atividade agropecuária, em especial a pecuária e a soja. Dessa maneira, os autores complementam que as políticas de combate ao desmatamento devem considerar quais são os fatores implícitos relacionados ao avanço de agropecuária no território da Amazônia.

De forma similar à Rivero et al. (2009), Diniz et al. (2009) buscaram identificar quais as principais causas do desmatamento na Amazônia legal brasileira entre 1997 e 2006 . Por meio de modelos dinâmicos para dados em painel, verificou-se a relação causal bidirecional entre desmatamento e as atividades de lavoura, tanto permanente, quanto temporária, com o tamanho do rebanho bovino e com o montante de crédito agrícola. Em relação à produção econômica, destaca-se a existência de relação unidirecional de causalidade entre o Produto Interno Bruto per capita e o desmatamento. Os autores afirmam que os resultados corroboram a hipótese de que o crescimento da atividade econômica causa um aumento na pressão sobre o desmatamento.

Ferreira, Venticinque e Almeida (2005), por meio da análise dados de desmatamento da INPE, examinam o desmatamento dentro e fora de áreas protegidas entre 2000 e 2003. Quanto à distribuição espacial do desmatamento, os resultados se assemelham aos resultados de Rivero et al., (2009). Contudo, nota-se a importância das Unidades de Conservação e Terras Indígenas na conservação da floresta Amazônica. O desmatamento nessas áreas foi de dez a vinte vezes menor do que em áreas contiguas sem proteção.

Ao analisar os resultados das pesquisas apresentadas nessa seção, considerando sua cronologia, sugere-se que o processo de desmatamento se expande, principalmente, nas fronteiras da floresta amazônica, progredindo para seu interior. Parte da margem Sul e Leste, apontada por Skole e Tucker (1993) como área de intenso desmatamento entre 1977 e 1988, corresponde à área fronteira consolidada de desmatamento, citada por Rivero et al,. (2009), ao analisar o desmatamento de 2000 a 2006.

Como abordado na introdução, a complexidade da dinâmica do desmatamento na Amazônia e a persistência do desmatamento ao longo do tempo são desafios a serem enfrentados. O presente estudo visa formar conhecimento quanto à dispersão espacial do desmatamento e sua relação com a agropecuária em um período mais recente, entre 2007 e 2017. Desse modo, sua contribuição é complementar aos demais estudos.

\section{Metodologia}

Nesta seção serão apresentados detalhes quanto ao método aplicado, a AEDE, os dados utilizados, com suas respectivas fontes, e os procedimentos adotados.

Análise exploratória de dados espaciais

A AEDE tem como objetivo descrever e visualizar a distribuição espacial, os padrões de associação espacial e a identificação de diferentes regimes espaciais (ALMEIDA, 2012). 
Para se implementar a AEDE se faz necessária a adoção de uma matriz de ponderação espacial (W), a qual consiste em uma matriz quadrada de dimensão $n \times n$, que expressa um arranjo espacial das interações resultantes do fenômeno a ser estudado (ALMEIDA, 2012).

$\mathrm{O}$ critério da contiguidade considera como vizinhas regiões com fronteira física comum. Regiões vizinhas recebem valor 1 , caso contrário, recebem valor 0 . Ao considerar apenas fronteiras físicas podem ocorrer erros de medida, devido ao fato de o mapa ser uma representação abstrata da configuração geográfica. Considerando a possibilidade desses erros, convenções de contiguidade devem ser adotadas, sendo elas Rainha (queen), Torre (rook) e Bispo (Bishop). A Figura 1 ilustra as convenções citadas.

Figura 1 - Convenções de Contiguidade Espacial.

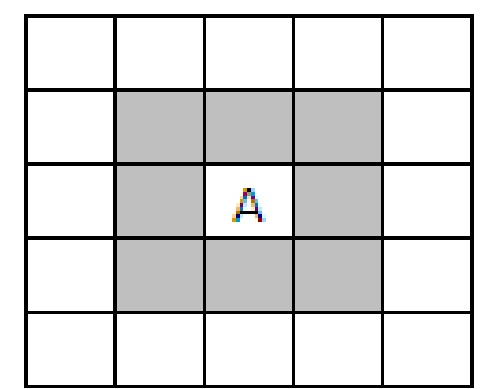

RAINHA

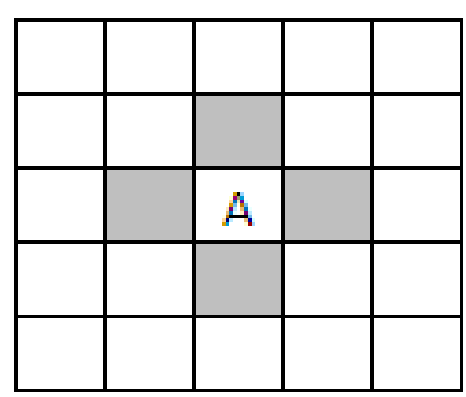

TORRE

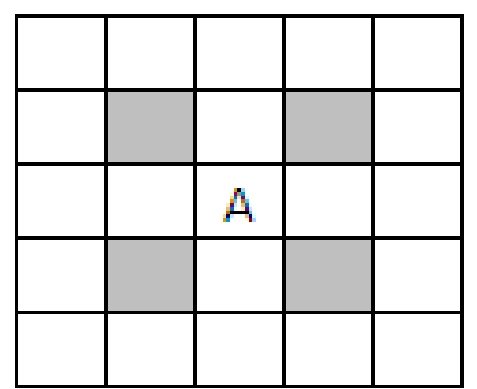

BISPO

Fonte: Almeida (2012).

Org: Elaborado pelos autores.

A convenção Rainha, além das fronteiras físicas, considera os vértices como contíguos. A convenção Torre considera apenas as fronteiras físicas. A convenção bispo considera apenas os vértices como vizinhos. As convenções rainha e Torre são as mais utilizadas na literatura pertinente.

O presente estudo utilizará da convenção Rainha, que considera, além das fronteiras físicas, os vértices como contíguos. A escolha da convenção Rainha é justificada pelo fato de o mapa ser uma representação abstrata da configuração geográfica. Destarte, a definição de vizinhança apenas pela existência de uma fronteira física pode não ser a melhor indicador de vizinhança.

Correlação espacial univariada e bivariada

A correlação espacial univariada global tem como objetivo verificar a existência, ou a inexistência, da aleatoriedade espacial, ou seja, se os valores de determinada variável de uma região não dependem dos valores da mesma variável em regiões vizinhas. Para verificar a aleatoriedade espacial, será aplicada a estatística I de Moran Global Univariada, a qual pode ser expressa por:

$$
I=\frac{n}{S_{0}} \frac{Z^{\prime} W z}{Z^{\prime} Z}
$$

Onde: $\mathrm{n}$ é o número de microrregiões; $z$ é variável de interesse padronizada; $W z$ são os valores médios da variável de interesse padronizada nos vizinhos; $n$ é o número de observações; e $S_{0}$ é o somatório dos elementos da matriz $W$. 
Segundo Almeida (2012), o I de Moran Global é um coeficiente de correlação espacial que resulta da relação entre a autocovariância dos produtos cruzados $\left(z^{\prime} W z\right)$ e pela variância dos dados $\left(z^{\prime} z\right)$.

Os indicadores de correlação espacial univariada variam de -1 a 1 e devem ser interpretados do seguinte modo: valores negativos indicam correlação negativa - As regiões apresentam valores altos, na variável observada de interesse, e seus vizinhos valores baixos, ou vice e versa; Valores positivos indicam correlação positiva: As regiões que apresentam valores altos, na variável observada de interesse, possuem vizinhos com a mesma característica. Valores próximos de 0 indicam ausência de correlação espacial.

A análise univariada se limita a apenas uma variável. Para mensurar a correlação especial de duas variáveis, se faz necessário analisar os indicadores de correlação espacial bivariada.

De forma semelhante à correlação univariada, o I de Moran Global bivariado busca verificar a existência, ou a inexistência, de efeitos espaciais entre uma variável de interesse em determinada área, com outra variável das áreas vizinhas. Tal indicador pode ser expresso por

$$
I^{x y}=\frac{n}{S_{0}} \frac{x W y}{x x}
$$

Onde: $\mathrm{n}$ é o número de microrregiões; $x$ é variável de interesse padronizada; $W y$ são os valores médios da variável de interesse padronizada nos vizinhos; $n$ é o número de observações; e $S_{0}$ é o somatório dos elementos da matriz $W$.

Os indicadores de correlação espacial, tanto univariada, quanto bivariada, são interpretados de forma semelhante. Os indicadores de correlação espacial bivariada variam de -1 a 1 e devem ser interpretados do seguinte modo: valores negativos indicam correlação negativa - As regiões apresentam valores altos, na variável observada $x$, e seus vizinhos valores baixos na variável $y$, ou vice e versa; Valores positivos indicam correlação positiva: As regiões que apresentam valores altos, na variável observada $x$, possuem vizinhos com a mesma característica, porém, na variável $y$.

A correlação global considera um valor para o conjunto de dados, contudo esse indicador pode esconder especificidades de cada região (ALMEIDA, 2012). A estatística I de Moran Local considera o grau de correlação individual de cada região.

O I de Moran Local é uma decomposição do I de Moran Global, onde a soma dos indicadores locais deve ser igual ao valor do I de Moran Global. O I de Moran Local é expresso matematicamente por:

$$
I_{i}=z_{i} \sum_{j=1}^{j} W_{i j} z_{j}
$$

Onde: $z_{i}$ é variável de interesse padronizada na observação $\mathrm{i} ; W_{i j}$ representa o elemento da matriz de ponderação espacial; $z_{j}$ é a variável de interesse padronizada na observação j.

Para facilitar a análise I de Moran Local, é sugerido que seus resultados sejam mapeados por meio da significância estatística dos resultados encontrados, chamado de mapa de Clusters LISA (Local Indicator of Spatial Association ). Neste mapa serão expostos quais localidades apresentaram I de Moran Local estatisticamente significante e qual seu tipo de associação espacial.

A associação espacial pode ser Alto-Alto (AA), quando a variável observada com valor alto é acompanhada de vizinhança com a mesma característica, Baixo-Baixo (BB), quando a variável observada com valor baixo é acompanhada de vizinhança com a mesma característica, Alto-Baixo (AB), quando a variável observada com valor alto acompanhada de 
vizinhança com valor baixo, e Baixo-Alto (BA), caso oposto da AB (ANSELIN, 1996). Quanto à análise bivariada, deve-se considerar a relação de uma variável observada em relação à outra variável de interesse de seus vizinhos.

Fonte dos dados e descrição das variáveis

Buscou-se demonstrar a variação da área de lavoura, do rebanho efetivo de pecuária bovino $^{2}$ e da taxa de desmatamento dos municípios da Amazônia legal brasileira. Os dados relativos à área de lavoura e do efetivo de pecuária foram coletados da Pesquisa de Pecuária Municipal de 2017 e Produção Agrícola Municipal de 2017, ambas realizadas pelo Instituto Brasileiro de Geografia e Estatística (IBGE). Os dados referentes ao desmatamento foram coletados do Projeto de Monitoramento de Desmatamento na Amazônia Legal por Satélite (PRODES), realizado pelo Instituto Nacional de Pesquisas Espaciais (INPE), referente aos anos de 2007 e 2017.

O Quadro 1 apresenta as variáveis de análise.

Quadro 1- Variáveis Utilizadas na Pesquisa

\begin{tabular}{|c|c|}
\hline Variável & Expressão Matemática \\
\hline Variação Percentual na Área de Lavoura & $\left(\frac{\text { Área de Lavoura em } 2017}{\text { Área de Lavoura em } 2007}\right)-1$ \\
\hline Variação Percentual no Efetivo de Pecuária & $\left(\frac{\text { Efetivo de Pecuária em } 2017}{\text { Efetivo de Pecuária em } 2007}\right)-1$ \\
\hline Variação Percentual na Área de Desmatamento & $\left(\frac{\text { Área de Desmatamento em 2017 }}{\text { Área de Desmatamento } m 2007}\right)-1$ \\
\hline
\end{tabular}

Fonte: Elaborado pelos autores.

Dentre os 772 municípios da Amazônia legal brasileira, 17 foram excluídos por não apresentar todos os dados necessários disponíveis. Além da exclusão de determinados municípios, foi necessário agregar os dados do município de Mojuí dos Campos (PA) aos dados de Santarém (PA), devido ao seu desmembramento e emancipação em 2009. Em relação ao tratamento dos mapas, os municípios excluídos foram removidos do shapefile, enquanto o município de Mojuí dos Campos foi agregado ao território de Santarém. Dessa maneira, foram consideradas 754 observações válidas.

De modo sucinto, realizaram-se os seguintes procedimentos: Definição do número de observações; Cálculo das variáveis para cada observação; Análise da variação da área de lavoura, pecuária e desmatamento; Estimativa do I de Moran global univariado; Análise dos Clusters de I de Moran local univariado; Estimativa do I de Moran global bivariado: Análise dos Clusters de I de Moran local bivariado

\section{Resultados}

Inicialmente, analisou-se a distribuição espacial da variação percentual da área de lavoura, do efetivo de pecuária e do desmatamento. Em seguida, fez-se a análise da autocorrelação univariada das variáveis, com a finalidade de verificar a presença, ou não, de concentração espacial. Por fim, analisou-se a autorrelação bivariada, com o intuito de demonstrar possíveis efeitos espaciais entre o avanço do desmatamento e a área de lavoura, bem como o avanço do desmatamento e a atividade pecuária. 
Variação da lavoura, pecuária e o desmatamento

Ao analisar a variação da área de lavoura nos municípios da Amazônia brasileira, verifica-se um aumento de 4,82\%, entre 2007 e 2017. Contudo, salienta-se que o avanço da área de lavoura não ocorreu de forma espacialmente homogênea. De acordo com a Mapa 2, constata-se que o avanço da área de lavoura ocorreu de forma mais intensa no nordeste do Mato Grosso, sudoeste e leste do Tocantins, sul do Maranhão, ao norte do Pará e sudeste do Amapá. Em contrapartida, verifica-se a redução da área de lavoura de forma mais dispersa, em especial no Maranhão, Pará, Rondônia e Amazonas.

Mapa 2- Variação Percentual da Área de Lavoura e do Efetivo de Pecuária.

a) Variação Percentual da Área de Lavoura

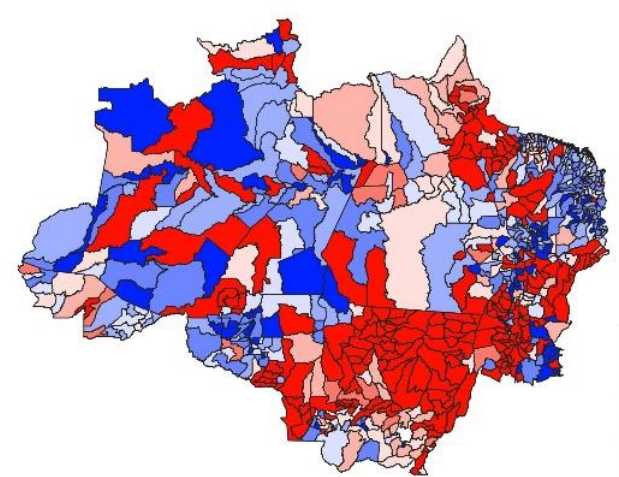

b)Variação Percentual do Efetivo de Pecuária

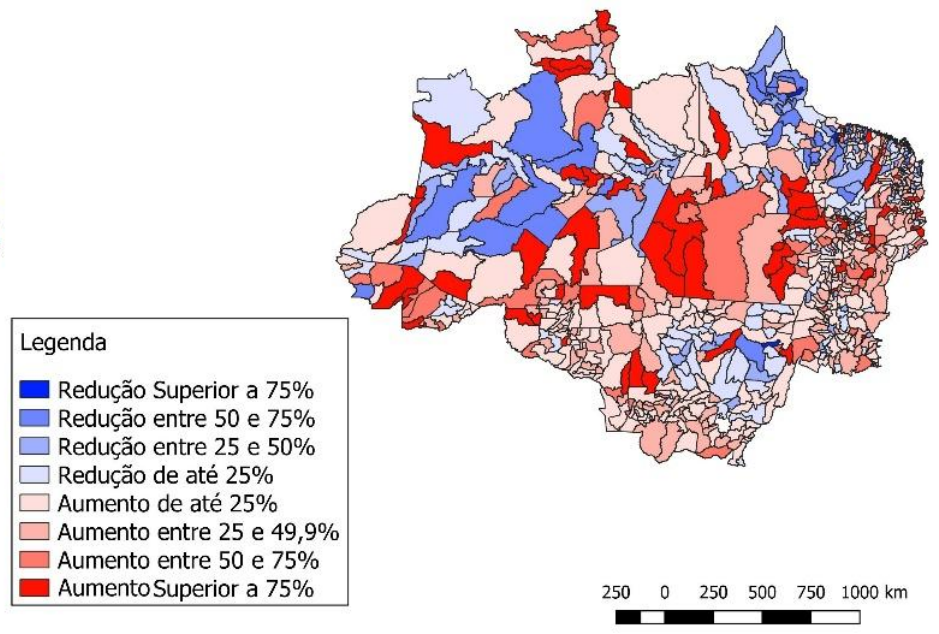

Fonte: Resultados da pesquisa.

Org: Elaborado pelos autores.

Em relação à variação do efetivo de pecuária, constata-se o aumento de $26,35 \%$ entre 2007 e 2017, representando um possível aumento da área aplicada à pecuária. Verifica-se que o crescimento do efetivo de pecuária ocorreu de forma mais homogênea em relação à atividade de lavoura. Contudo, destaca-se a maior intensidade do crescimento no oeste do Pará, centro do Acre e parte de Roraima. Constata-se, também, a redução do efetivo de pecuária em parte do Amazonas e parte do Amapá.

Ao analisar o avanço do desmatamento, verifica-se aumento de 8,93\% na área desmatada. Conforme o Mapa 3, nota-se a existência de uma faixa de desmatamento zero e de baixo desmatamento, composta por Rondônia, Mato Grosso, Tocantins e Maranhão, contígua à uma faixa de maior desmatamento, composta pelo Acre, Amazonas, Pará e Amapá. Verifica-se, também, o avanço significativo do desmatamento em Roraima.

A faixa de baixo desmatamento, localizada em contiguidade à de alto desmatamento, abrange parte da fronteira consolidada do desmatamento, apresentada por Rivero et al., (2009), e parte da área da Amazônia legal brasileira com bioma de cerrado. Desse modo, não pode ser interpretada como um indicativo de maior preservação ambiental, mas de degradação ambiental. Em contrapartida, a faixa de alto desmatamento pode ser interpretada como a fronteira de desmatamento da Amazônia brasileira.

A variação do desmatamento pode ser interpretada como a continuidade do processo de progressão em direção ao seu interior, ao comparar os resultados com os trabalhos de 
Skole e Tucker (1993), Kampel, Câmara e Quintanilha (2000), Rivero et al., (2009), Ferreira, Venticinque e Almeida (2005). De modo semelhante, a expansão do desmatamento em Roraima, possivelmente, está associada a um efeito de transbordamento, visto que Kampel, Câmara e Quintanilha (2000) identificaram a presença de avanço de desmatamento em alguns municípios de Roraima, apesar de não apresentar concentração espacial significante.

Mapa 3 - Variação Percentual da Área de Desmatamento.

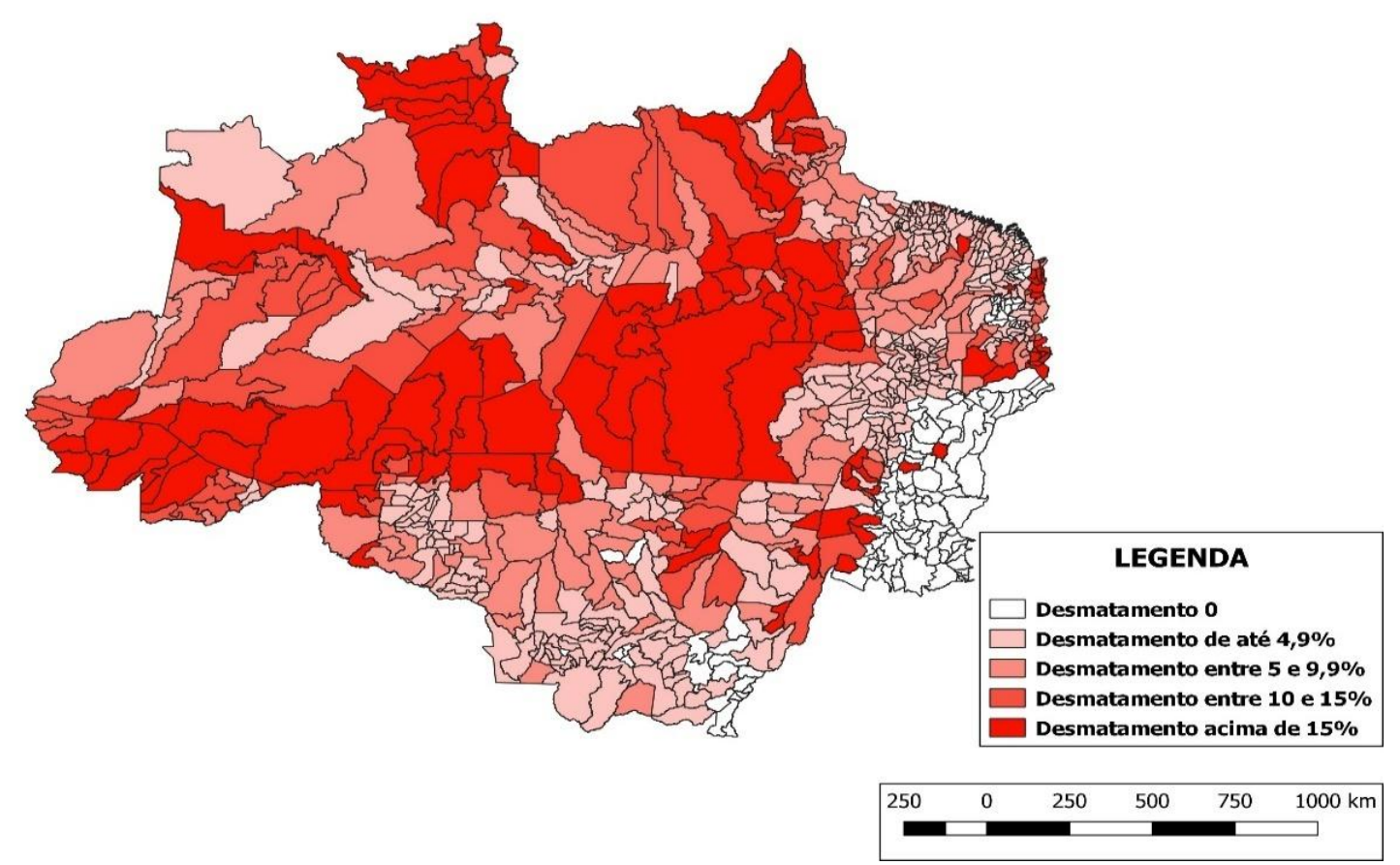

Fonte: Resultados da pesquisa.

Org: Elaborado pelos autores.

Correlação espacial univariada

Apesar da análise da distribuição espacial oferecer indícios quanto à concentração espacial, é somente por meio da aplicação de métodos adequados, como o I de Moran, que se pode constatar a presença, ou ausência, de concentração espacial.

Verifica-se a existência de correlação espacial estatisticamente significante na variação da área de desmatamento, conforme Tabela 1. O I de Moran Global foi de 0,391, com significância estatística superior à $99 \%$.

Tabela 1 - I de Moran Global.

\begin{tabular}{cccc}
\hline Variável & I de Moran Global & Pseudo P-value & Permutações \\
\hline Lavoura & 0,298 & 0,001 & 999 \\
Pecuária & 0,018 & 0,075 & 999 \\
Desmatamento & 0,391 & 0,001 & 999 \\
\hline
\end{tabular}

Fonte: Elaborado pelos autores.

Tal resultado indica correlação espacial positiva menos intensa que nos períodos de 1991 a 1992 e 1992 a 1994, apresentadas por Kampel, Câmara e Quintanilha (2000), corroborando os indícios de concentração espacial do processo de desmatamento, mencionado 
por Skole e Tucker (1993). Salienta-se a necessidade de mais estudos para inferir quanto à evolução da tendência de desconcentração espacial do desmatamento.

Verifica-se a existência de correlação espacial na variação da área de lavoura, com I de Moran de 0,298. No tocante à pecuária, apesar do I de Moran global sugerir a inexistência de autorrelação espacial, observa-se que o indicador apresentou significância estatística inferior a 0,050. Destaca-se, ao analisar o I de Moran, a maior concentração espacial do desmatamento, em relação à variação da área de lavoura.

O I de Moran Global indica a presença, ou ausência, de concentração espacial para o conjunto de dados. Para verificar a formação de clusters espaciais de forma local, foi empregada a estatística I de Moran Local, cujos valores foram dispostos no mapa de cluster LISA.

No tocante à variação da área de lavoura, foram identificados três clusters de padrão Alto-Alto, e cinco clusters de padrão Baixo-Baixo, conforme o Mapa 4. Em relação à variação da pecuária, foram identificados nove clusters, sendo dois de padrão Alto-Alto e sete de padrão Baixo-Baixo. No tocante à variação do desmatamento, foram identificados seis clusters de padrão Alto-Alto e seis clusters de padrão Baixo-Baixo.

Mapa 4 - Clusters de I de Moran Local Univariado.

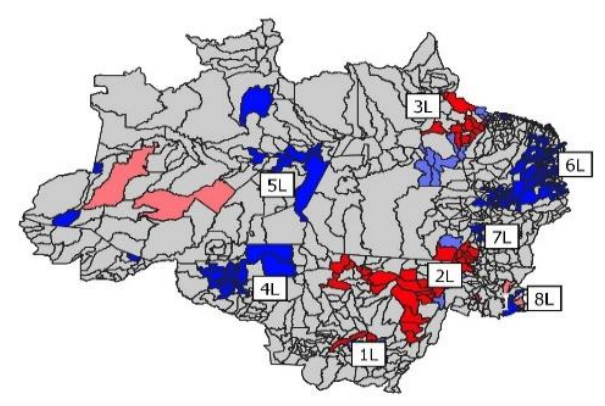

a)Variação Percentual da Área de Lavoura

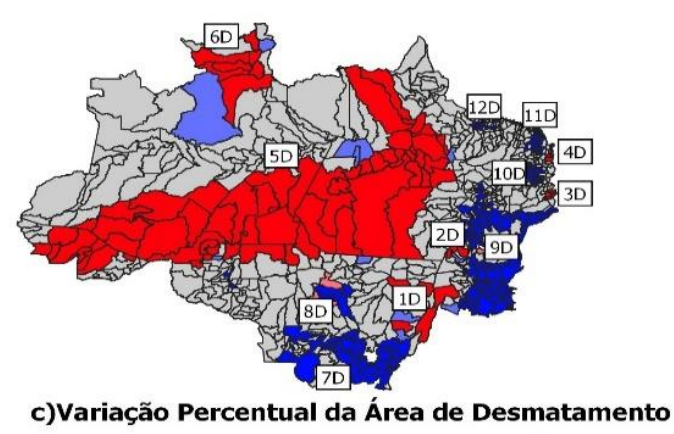

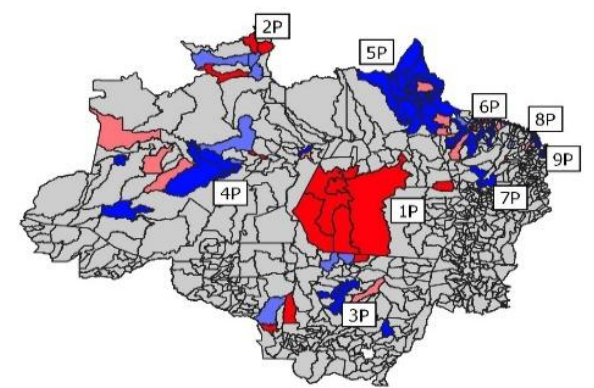

b)Variação Percentual do Efetivo de Pecuária $250 \quad 0 \quad 2505007501000 \mathrm{~km}$

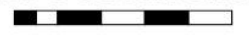

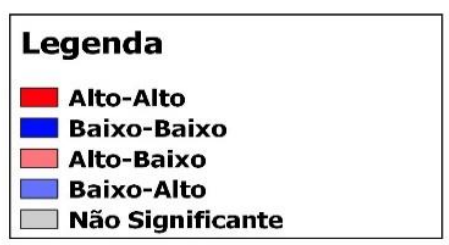

Fonte: Resultados da pesquisa.

Org: Elaborado pelos autores.

Os clusters 1D, 2D, 3D e 4D², de padrão Alto-Alto, estão localizados no sudeste e leste da Amazônia brasileira. Notadamente, são clusters com menor dimensão, em relação aos demais clusters do mesmo padrão, e estão localizados entre clusters de baixo desmatamento, representando uma possível extração da floresta remanescente em áreas de desmatamento consolidado.

O Cluster 5D, que abrange uma faixa de território de parte do Acre, sul do Amazonas, sudoeste, centro e norte do Pará, e sudoeste do Amapá, e o Cluster 6D, no Tocantins, representam avanço do desmatamento. Salienta-se que o Cluster 5D pode ser interpretado 
como possível avanço da fronteira, ou arco do desmatamento, abordados por Rivero et al., (2009) e Kampel, Câmara e Quintanilha (2000). Além do avanço do desmatamento, o cluster 6D indica a consolidação de uma nova fronteira de avanço do desmatamento, entretanto, interna à Amazônia.

Quanto aos clusters do padrão Baixo-Baixo, 7D, 8D, 9D, 10D, 11D e 12D, estão localizados na faixa de sul e leste da Amazônia. Salienta-se que não foram identificados clusters de Baixo-Baixo dentro das fronteiras do desmatamento. Mais uma vez, destaca-se que tais cluster, possivelmente, não representam a conservação da floresta amazônica, mas justamente o oposto.

Ao comparar os clusters identificados nesse estudo, com os resultados de Kampel, Câmara e Quintanilha (2000), novos indícios da interiorização do processo de desmatamento são evidenciados. Destaca-se a redução da participação dos municípios de Rondônia e do Mato Grosso nos clusters de alto desmatamento, em detrimento do aumento de municípios no Amazonas.

No tocante à relação entre os clusters de desmatamento e lavoura, verifica-se que os clusters 1D, 2D e 2L, todos de padrão Alto-Alto, apresentam municípios em comum, indicando uma possível relação positiva entre desmatamento e lavoura ao nordeste do Mato Grosso e ao sudoeste do Tocantins. De modo contrário, a coexistência espacial dos clusters 5L, Baixo-Baixo, e 5D, Alto-Alto, provavelmente indica a inexistência de relação entre desmatamento e lavoura no centro da Amazônia.

Quanto à relação entre os clusters de desmatamento e pecuária, a coexistência espacial dos clusters 6D e 2P, em Roraima, e dos clusters 1P e 5D, ao sudoeste do Pará, todos de padrão Alto-Alto, indicam uma possível relação entre pecuária e desmatamento nessas regiões. Contudo, ressalta-se que tais inferências são indícios, necessitando de métodos adequados para confirmação, como aplicado e verificado na sequência.

Correlação espacial bivariada

Como exposto anteriormente, na metodologia, o I de Moran bivariado é utilizado para constatar a presença de correlação espacial entre a variação percentual do desmatamento de determinado município e a variação percentual da área de lavoura, além da variação do efetivo de pecuária dos municípios vizinhos. Destaca-se que a presença de correlação espacial bivariada não pode ser interpretada como sentença de causa e efeito. Para comprovar a relação de causa e efeito, é necessário que se empreguem métodos apropriados. Contudo, a presença de correlação provê indícios significantes da relação entre as variáveis analisadas.

Os indicadores de I de Moran Bivariado Global do desmatamento em relação à variação na atividade de lavoura e do efetivo de pecuária, são apresentadas na Tabela 2.

Tabela 2 - I de Moran Bivariado Global.

\begin{tabular}{cccc}
\hline Variável & $\begin{array}{c}\text { I de Moran Bivariado } \\
\text { Global }\end{array}$ & Pseudo P-value & Permutações \\
\hline Desmatamento e Lavoura & 0,051 & 0,003 & 999 \\
Desmatamento e Pecuária & 0,017 & 0,105 & 999 \\
\hline
\end{tabular}

Fonte: Elaborado pelos autores.

Ao analisar, o I de Moran bivariado global, constata-se a inexistência de correlação espacial entre a variação no desmatamento e a área de lavoura. No tocante à pecuária, os dados são inconclusivos. Apesar do I de Moran global bivariado indicar a inexistência de 
correlação espacial entre a variação do desmatamento e a variação no efetivo de pecuária, a significância estatística foi superior a 0,050 .

Assim como na análise univariada, o mapeamento dos resultados I de Moran local permite identificar a formação de clusters espaciais entre as variáveis analisadas. Em relação ao desmatamento e lavoura, foram identificados quatro clusters de padrão Alto-Alto, e seis clusters de padrão Baixo-Baixo, conforme o Mapa 5. Quanto aos Clusters Alto-Alto, foram denominados: Cluster 1DL - no nordeste do Mato Grosso e sudoeste do Tocantins, composto por seis municípios; Cluster 2DL - no oeste do Tocantins, composto por sete munícipios; Cluster 3 DL - no leste do Pará, composto por três municípios; e Cluster 4DL - no nordeste do Pará, composto por quatro municípios;

Mapa 5 - Clusters de I de Moran Local Bivariado.

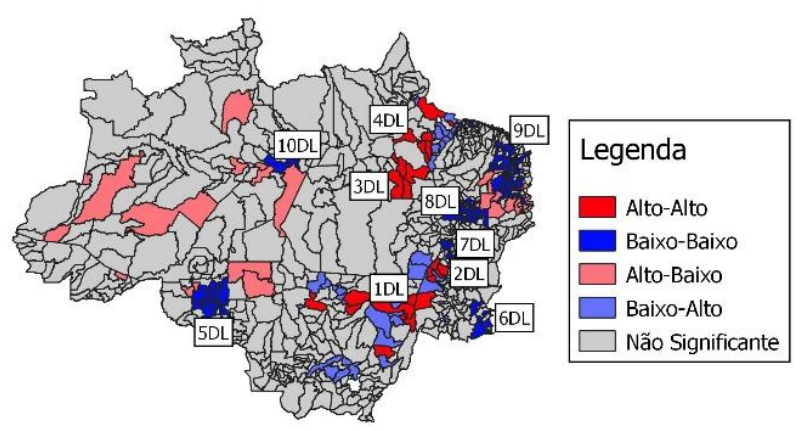

a) Desmatamento e Lavoura
$250 \quad 0 \quad 2505007501000 \mathrm{~km}$

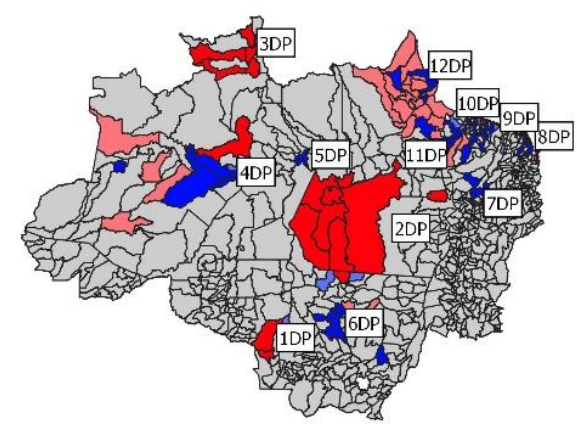

b) Desmatamento e Pecuária

Fonte: Resultados da pesquisa.

Org: Elaborado pelos autores.

No tocante aos clusters Baixo-Baixo, foram denominados: Cluster 5DL - no centro de Rondônia, composto por dezenove municípios; Cluster 6DL - no sudeste do Tocantins, composto por seis municípios; Cluster 7DL - no centro do Tocantins, composto por cinco municípios; Cluster 8DL - no norte do Tocantins, e sudoeste do Maranhão, composto por vinte e cinco municípios; Cluster 9DL - no nordeste do Maranhão, composto por cinquenta e quatro municípios; e Cluster 10DL - no leste do Amazonas, composto por 3 municípios.

Constata-se que a existência dos clusters 1DL e 2DL, ambos de padrão Alto-Alto, corrobora para a existência de relação entre desmatamento e pecuária. Cabe enfatizar que esses clusters estão localizados em áreas que não pertencem à fronteira do desmatamento, representada pelo cluster 5D. Em contrapartida, os clusters 3DL e 4DL, ambos de padrão Alto-Alto, demonstram a existência de correlação espacial entre desmatamento e lavoura, ao leste e ao nordeste do Pará.

Os clusters 5DL, 6DL, 7DL, 8DL e 9DL, de padrão Baixo-Baixo, estão localizados em áreas de desmatamento consolidado. Sendo assim, sua existência, possivelmente, está associada à retração da lavoura, dada a restrição do avanço do desmatamento. Em contraponto, o cluster 10DL, de padrão Baixo-Baixo, está localizado no centro do Amazonas, possivelmente representando uma área ainda não explorada de forma significativa.

Em relação ao desmatamento e ao efetivo de pecuária, foram identificados três clusters de padrão Alto-Alto, e nove clusters de padrão Baixo-Baixo. Quanto aos clusters Alto-Alto, foram denominados: Cluster 1DP - no oeste do Mato Grosso, composto por dois municípios; Cluster 2 DP - no sudoeste do Pará e no norte do Mato Grosso, composto por sete municípios; Cluster 3DP - localizado em Roraima, composto por cinco municípios. 
No tocante aos clusters de padrão Baixo-Baixo, foram denominados: Cluster 4DP no centro do Amazonas, composto por três municípios; Cluster 5DP - no leste do Amazonas, composto por dois municípios; Cluster 6DP - no centro do Mato Grosso, composto por cinco municípios; Cluster 7DP - no leste do Pará e oeste do Maranhão, composto por três municípios; Cluster 8DP - no nordeste do Maranhão, composto por oito municípios; Cluster 9DP - no noroeste do Maranhão, composto por dez municípios; Cluster 10DP - no noroeste do Maranhão, composto por dois municípios; Cluster 11DP - no nordeste do Pará, composto por sete municípios; Cluster 12DP - no Amapá, composto por três municípios.

Verifica-se que os Clusters 1DP, no oeste do Mato Grosso, e Cluster 3DP, no Roraima, ambos de padrão Alto-Alto, não estão localizados na fronteira do desmatamento. Em contraponto, os clusters 2DP, de padrão Alto-Alto, estão localizados em áreas de fronteira do desmatamento. Assim, como na atividade de lavoura, verifica-se que a correlação entre o avanço do desmatamento e a pecuária não se limita às áreas de fronteira agrícola.

A existência dos clusters 4DP e 5DP, de padrão Baixo-Baixo, assim como o cluster 10DL, possivelmente está associada à baixa exploração dessas áreas. Em relação aos demais clusters de padrão Baixo-Baixo, indicam a correlação de redução da atividade de pecuária, acompanhada de ínfimo aumento do desmatamento, sendo localizados em áreas externas à fronteira do desmatamento.

De maneira generalizada, verifica-se a presença de concentração espacial no processo de desmatamento. Quanto à distribuição espacial do desmatamento, verifica-se que o processo de desmatamento avançou sobre as antigas fronteiras. Os resultados apresentados corroboram a relação entre as atividades agropecuárias citadas por Rivero et al. (2009), Diniz et al. (2008), Andrade e Chagas (2017) e Diniz et al. (2009). Contudo, indicam que os impactos não são espacialmente distribuídos de forma homogênea.

\section{Considerações Finais}

O presente estudo buscou analisar a distribuição e a concentração espacial do desmatamento e das atividades agropecuárias, representadas pela área de lavoura e o efetivo de pecuária, nos municípios da Amazônia legal entre 2007 e 2017.

Os resultados indicam que o avanço do desmatamento ocorreu de forma mais intensa em uma faixa de área que abrange o Acre, Sul do Amazonas, Pará e Amapá, e em Roraima. Quanto às áreas de baixo desmatamento, foram localizadas em uma faixa Sul e Leste da Amazônia. Áreas estas, caracterizadas por estudos antecedentes como de desmatamento consolidado.

Por meio da análise do I de Moran global, constatou-se a existência de concentração espacial do desmatamento. Quanto aos clusters de padrão Alto-Alto, verifica-se a existência de clusters contíguos às áreas de desmatamento consolidado, representando as novas fronteiras de desmatamento. O Cluster 5D pode ser interpretado como um novo arco do desmatamento, resultado do avanço sobre as antigas fronteiras. O cluster 6D representa uma possível nova fronteira, entretanto, interna à fronteira de desmatamento da Amazônia.

Entre o Cluster 5D e 6D, não foram identificados clusters de baixo desmatamento, sinalizando indícios de presença de desmatamento nessa área. Acrescenta-se que os clusters de baixo desmatamento foram localizados em áreas de desmatamento consolidado. Deste modo, não se pode associar os clusters de baixo desmatamento à preservação da Amazônia.

De forma global, identificou-se a inexistência de correlação espacial entre a variação da área de desmatamento e a variação na área de lavoura. Em relação ao efetivo de pecuária, os resultados indicam uma possível inexistência de correlação espacial, contudo o indicador 
não apresentou significância estatística. Entretanto, ao analisar de forma local, foram identificadas áreas com presença de correlação estatisticamente significante, caracterizados como clusters. A presença dos clusters de padrão Alto-Alto, corroboram o prognóstico de pressão ambiental, advindo as atividades agropecuárias, em especial da pecuária, conforme a revisão de literatura. Porém, salienta-se que tal impacto não ocorre de forma espacialmente homogênea.

Verifica-se a presença de clusters Alto-Alto, tanto em áreas de fronteira do desmatamento, como os clusters 3DL, 4DL e 2DP, quanto em áreas de não fronteira, como os clusters 1DL, 2 DL e 3DP. Os clusters 5D e 6D indicam possíveis fronteiras do desmatamento. Destaca-se que o cluster 6D indica o surgimento de uma fronteira de desmatamento interna à Amazônia. A coexistência espacial dos clusters 3DP e 6 DP aponta indícios do possível impacto da pecuária sobre o avanço do desmatamento.

De maneira geral, os resultados indicam o avanço do desmatamento sobre as fronteiras existentes, bem como pela consolidação de novas, como em Roraima. Dado o caráter multifacetado da dinâmica do desmatamento, sugere-se, como estudos futuros, analisar os efeitos espaciais de fatores socioeconômicos, como a atividade agropecuária, o nível e a distribuição de renda, a ocupação demográfica, a atividade econômica e demais fatores relacionados pela literatura acerca do tema.

\section{Notas}

${ }^{1}$ A Pesquisa de Pecuária Municipal não apresenta a área destinada à pecuária. Dessa maneira, utilizou-se do número de rebanho efetivo de pecuária como forma de representar a variação da área da atividade de pecuária. Optou-se pelo bovino por ser o principal tipo de criação da região.

${ }^{2}$ Utilizou-se das letras D, L, P para indicar desmatamento, lavoura e pecuária, respectivamente.

\section{Referências}

ALENCAR, Ane. et al. Desmatamento na Amazônia: indo além da "emergência crônica". Belém: Ipam, 2004. Não paginado.

ALMEIDA, Eduardo. Econometria espacial aplicada. 1.ed. Campinas: Alínea, 2012. 498 p.

ANSELIN, Luc. The Moran Scatterplot as an ESDA tool to asses local instability in spatial association, in: FISCHER, M.; SCHOLTEN, H. K.; UNWIN, D. (Eds), Spatial Analitycs Perspectives on GIS, Londres: Taylor \& Francis, 1995. 256 p. p. 121-138.

ASSUNÇÃO, Juliano; GANDOUR, C. C.; ROCHA, R. A queda do desmatamento na Amazônia brasileira: preços ou políticas. Climate policy initiative. Rio de Janeiro: Núcleo de Avaliação de Políticas Climáticas, PUC-Rio, 2015.

BACHA, Carlos José Caetano. O Uso de Recursos Florestais e as Políticas Econômicas Brasileiras - Uma Visão Histórica e Parcial de um Processo de Desenvolvimento. Estudos Econômicos, São Paulo, v. 34, n.2, p. 393-426, 2004.

BRASIL. Plano Amazônia Sustentável: Diretrizes para o desenvolvimento sustentável da Amazônia brasileira. Brasília: MMA, 2008. Disponível em: < http://www.mma.gov.br/estruturas/sca/_arquivos/plano_amazonia_sustentavel.pdf $>$. Acesso em: 10 nov. 2018. 
Serviços Florestal Brasileiro. Sistema Nacional De Informações Florestais (SNIF) 2018. Disponível em: < http://snif.florestal.gov.br/pt-br/ >. Acesso em: 10 nov. 2018.

BROOK, Barry William.; SODHI, Navjot S.; NG, Peter Kee Lin. Catastrophic extinctions follow deforestation in Singapore. Nature, [s.1], v. 424, n. 6.947, p. 420, jul. 2003

CHAGAS, André Luiz Squarize; ANDRADE, Luiza Cardoso de. Custo de oportunidade da preservação ambiental: o caso da Amazônia Legal Brasileira. Revista Brasileira de Estudos Regionais e Urbanos, [s.1.], v. 11, n.3, p. 384-405, 2017.

COSTA, Simone S. Thomazi. Introdução à economia do meio ambiente. Análise, Porto Alegre, v. 16, n. 2, p. 301-323, ago./dez. 2005.

DINIZ, Marcelo Bentes; DINIZ, Márcia Jucá Teixeira. Exploração dos recursos da biodiversidade da Amazônia Legal: uma avaliação com base na abordagem do Sistema Nacional/Regional de Inovação. REDES: Revista do Desenvolvimento Regional, Santa Cruz do Sul, v. 23, n. 2, p. 210-237, mai./ago.2018.

DINIZ, Marcelo Bentes. et al. A Amazônia (Legal) Brasileira: evidências de uma condição de armadilha da pobreza?. In: RIVERO, S.; JAYME JR., F. G.. (Org.). As Amazônias do Século XXI. 1ed.Belém: EDUFPA, 2008, v. 1, 292p. p. 125-154.

DULLEY, Richard Domingues. Noção de natureza, ambiente, meio ambiente, recursos ambientais e recursos naturais. Agric. São Paulo, São Paulo, v. 51, n. 2, p. 15-26, jul./dez. 2004.

FEARNSIDE, Philip. M. Deforestation in Brazilian Amazonia: history, rates, and consequences. Conservation biology, [s.1.], v. 19, n. 3, p. 680-688, jun. 2005.

FERREIRA, Leandro Valle; VENTICINQUE, Eduardo; ALMEIDA, Samuel. O desmatamento na Amazônia e a importância das áreas protegidas. Estudos Avançados, São Paulo, v. 19, n. 53, p. 157-166, jan./abr. 2005

HOMMA, Alfredo Kingo Oyama. Amazônia: os avanços e os desafios da pesquisa agrícola. Parcerias Estratégicas, Brasília, v. 18, n. 36, p. 33-54, jan/jun, 2013.

IBGE, Instituto Brasileiro de Geografia e Estatística Pesquisa de Pecuária Municipal PPM 2017. Disponível em: < https://sidra.ibge.gov.br/pesquisa/ppm/quadros/brasil/2017 >. Acesso em: 18 out. 2018

— Produção Agrícola Municipal - PAM 2017. Disponível em: <https://sidra.ibge.gov.br/pesquisa/pam/tabelas >. Acesso em: 18 out. 2018

INPE, Instituto Nacional de Pesquisas Espaciais. Projeto de Monitoramento de Desmatamento na Amazônia Legal por Satélite - PRODES. 2018. Disponível em: <http://www.dpi.inpe.br/prodesdigital/prodesmunicipal.php >. Acesso em: 18 out. 2018 
KAMPEL, Silvana Amaral; CÂMARA, Gilberto; QUINTANILHA, José Alberto. Análise exploratória das relações espaciais do desflorestamento na Amazônia legal brasileira. Instituto Nacional de Pesquisas Espaciais - INPE, São José dos Campos, 2000. Disponível em: < http://www.dpi.inpe.br/geopro/trabalhos/silvana_gisbrasil2000.pdf > . Acesso em: 18 out. 2018

MAHAR, Dennis J. Government policies and deforestation in Brazil's Amazon region. 1.ed. Washington: World Bank, 1989. 56 p.

MARGULIS, Sérgio. Causas do desmatamento da Amazônia brasileira. 1.ed. Brasília: Estação Gráfica, 2003. 100 p.

MEDVIGY, David; WALKO, Robert. L.; AVISSAR, Ron. Effects of deforestation on spatiotemporal distributions of precipitation in South America. Journal of Climate, [s.1.], v. 24, n. 8, p. 2147-2163, abr. 2011.

MELO, Andrea Sales Soares Aze. Economia dos recursos naturais e seus indicadores de escassez: uma questão de sustentabilidade. Análise Econômica, Porto Alegre, v. 23, n. 44, p. 131-146, set. 2005.

NEPSTAD, Daniel. et al. Slowing Amazon deforestation through public policy and interventions in beef and soy supply chains. Science, Washington, v. 344, n. 6188, p. 11181123, jun. 2014.

ROMEIRO, Ademar Ribeiro. Economia ou economia política da sustentabilidade? Texto para Discussão. IE/UNICAMP, Campinas, n. 102, set. 2001.

RIVERO, Sérgio; ALMEIDA, Oriana; ÁVILA, Saulo; OLIVEIRA, Wesley. Pecuária e desmatamento: uma análise das principais causas diretas do desmatamento na Amazônia. Nova Economia (UFMG. Impresso), Belo Horizonte, v. 19, p. 41-66, jan./abr. 2009.

SKOLE, David; TUCKER, Compton. Tropical deforestation and habitat fragmentation in the Amazon: satellite data from 1978 to 1988. Science, Washington v. 260, n. 5116, p. 19051910, jun. 1993.

SODHI, Navjot S. et al. Southeast Asian biodiversity: an impending disaster. Trends in ecology \& evolution, [s.1.], v. 19, n. 12, p. 654-660, dez. 2004.

SHUKLA, Jagadish; NOBRE, Carlos Afonso; SELLERS, Piers. Amazon deforestation and climate change. Science, Washington, v. 247, n. 4948, p. 1322-1325, 1990.

WORLD COMISSION ON ENVIRONMENT AND DEVELOPMENT - WCED. Our Common Future. [S.l.:s.n.] 1987. Disponível em: < https://ambiente.files.wordpress.com/2011/03/brundtland-report-our-common-future.pdf $>$. Acesso em: 20/mar/2019. 